\title{
Permanent atrial fibrillation in patients with a dual-chamber pacemaker
}

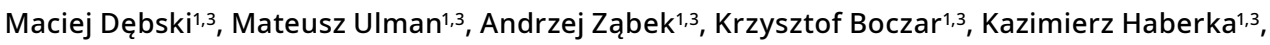 \\ Marcin Kuniewicz ${ }^{1-3}$, Jacek Lelakowski ${ }^{1,3}$, Barbara Małecka ${ }^{1,3}$ \\ 1 Department of Electrocardiology, John Paul II Hospital, Kraków, Poland \\ 2 Department of Anatomy, Jagiellonian University Medical College, Kraków, Poland \\ 3 Institute of Cardiology, Jagiellonian University Medical College, Kraków, Poland
}

KEY WORDS

atrial fibrillation, mortality, pacing, sex

\section{EDITORIAL}

by Boriani and Vitolo, see p. 1119
Correspondence to: Andrzej Ząbek, MD, PhD, MSc, Department of Electrocardiology, John Paul II Hospital, ul. Prądnicka 80, 31-202 Kraków, Poland, phone: +48126142381, email: andrzej_j_z@poczta.onet.pl Received: June 12, 2019. Revision accepted: September 16, 2019. Published online: September 17, 2019. Kardiol Pol. 2019; 77 (12): 1140-1146 doi:10.33963/KP.14974 Copyright by the Author(s), 2019

\section{ABSTRACT}

BACKGROUND Atrial fibrillation (AF) is thought to be a progressive arrhythmia. The impact of sex and position of right ventricular lead is not well recognized. Whilst nonparoxysmal AF compared with paroxysmal AF has been associated with increased mortality in the general population, its prognostic significance in patients with a dual-chamber (DDD) pacemaker is less clear.

AIMS The aim of the study was to determine the incidence of permanent AF in patients with a DDD pacemaker, analyze the effect of selected baseline characteristics on permanent AF development, and examine the impact of permanent AF on patient survival.

METHODS A retrospective cohort study included 3932 consecutive patients who underwent DDD pacing system implantation between 1984 and 2014. Follow-up was completed in August 2016. We included 3771 patients (96\%) with post-operative follow-up and known vital status. Occurrence of permanent AF and all-cause mortality were the study endpoints.

RESULTS During mean follow-up of 6.5 years, permanent AF occurred in 717 patients (19\%). Sex (hazard ratio [HR], 1.316; 95\% CI, 1.134-1.528, for men), age at implant (HR, 1.041; 95\% CI, 1.033-1.049, 1-year increase), history of AF (HR, 3.521; 95\% CI, 3.002-4.128) were independently associated with permanent AF development, whereas position of right ventricular lead (apical versus nonapical) and primary pacing indication (atrioventricular block versus sick sinus syndrome) were not related to permanent AF. Permanent AF was a significant risk factor for increased mortality (age- and sex-adjusted HR, 1.475; 95\% CI, 1.294-1.682). CONCLUSIONS Permanent AF occurrence was independently predicted by advanced age at implant, male sex, and preexisting AF and associated with worse survival.

INTRODUCTION Atrial fibrillation (AF) is the most commonly encountered arrhythmia in the population and has been referred to as "epidemic" affecting approximately $3 \%$ of adults. ${ }^{1}$ $\mathrm{AF}$ is considered to be a progressive arrhythmia. In many patients, it starts with short, infrequent episodes and over time progresses to longer and more frequent episodes. In a minority of patients, AF remains paroxysmal over long time or may even regress. In majority of patients, however, the burden of AF increases and becomes sustained. Persistent, long-standing AF is diagnosed if rhythm control strategy is adopted, and permanent $\mathrm{AF}$, if no interventions are undertaken to revert it to sinus rhythm. ${ }^{2}$ Importantly, chronic AF precludes the use of dual-chamber pacing due to inability to pace the atrium.

It is well established across many populations that $\mathrm{AF}$ is a risk factor for increased mortality. ${ }^{3-8}$ Of note, in our recent study, we demonstrated that preimplant AF was not associated with reduced survival in consecutive recipients of dual-chamber (DDD) permanent pacemaker (PPM). ${ }^{9}$ A retrospective analysis of patients implanted with modern DDD PPM capable of storing AF data showed that AF burden was not 


\section{WHAT'S NEW?}

The study revealed that male sex compared with female sex along with history of atrial fibrillation and advanced age were associated with increased risk of permanent atrial fibrillation. On the other hand, position of the right ventricular lead and pacing indication did not influence the occurrence of permanent atrial fibrillation. Furthermore, we demonstrated that in patients with dual-chamber pacemaker, permanent atrial fibrillation resulted in a significant reduction of survival.

predictive of mortality. ${ }^{10}$ On the other hand, a recent real-world community-based cohort study and a meta-analysis involving 6 large-scale randomized controlled trials on oral anticoagulation therapy demonstrated that patients with persistent or permanent $\mathrm{AF}$ compared with paroxysmal AF had significantly higher risk of all-cause mortality. ${ }^{11,12}$ In addition, the associations between development of permanent $\mathrm{AF}$ and baseline variables such as pacing indication, sex, and position of the right ventricular (RV) lead remain unclear. Previous reports suggested that the natural history of AF was affected by the cumulative percentage of atrial and ventricular pacing, therefore, outcomes in general populations may not be applicable to DDD paced cohorts. ${ }^{10,13} \mathrm{We}$ sought to determine risk factors favoring development of permanent $\mathrm{AF}$ and assess the effect of permanent $\mathrm{AF}$ on long-term survival in a large cohort with DDD pacing.

METHODS The study cohort consisted of all consecutive patients who underwent de novo DDD PPM implantation between October 4, 1984 and December 31, 2014 at a high-volume tertiary reference university cardiology center. Patients were followed until August 31, 2016 or death, whichever came first. Data on patients' vital status and dates of death were collected from the national death registration system after the follow-up period. The survival and lead-related complications in the present cohort have been studied previously. $9,14,15$ The endpoints were the onset of permanent $\mathrm{AF}$ and all-cause mortality. The data used in the analysis included 1) patients' demographic baseline characteristics: age at implantation and sex; 2) index arrhythmia (primary electrocardiographic pacing indication): atrioventricular block (AVB) or sick sinus syndrome (SSS); 3 ) a history of AF prior to DDD PPM implantation; 4) position of the RV lead: apical or nonapical at discharge from the department; 5) time of permanent AF onset; and 6) date of death declared in the death certificate. These data were retrospectively gathered from paper and electronic medical records from hospital admissions, operative reports, and cardiology clinic visits that included pacemaker checks. We retrospectively reviewed device interrogation reports, electrocardiograms (ECG), and Holter ECG recordings from inpatient and outpatient encounters. Patients had standard device interrogation follow-up approximately 3 months after implantation, followed by every 6 to 12 months thereafter. We did not distinguish between AF and atrial flutter. Preimplant AF was defined as AF documented on ECG prior to DDD PPM implantation and included paroxysmal and persistent $\mathrm{AF}$ provided that the restoration of sinus rhythm was planned after implantation. Patients with permanent $\mathrm{AF}$ were routinely implanted with single-chamber ventricular (VVI) PPM throughout the study period. We used the 2016 European Society of Cardiology guidelines to define permanent $\mathrm{AF}^{2}{ }^{2}$ In the present study the minimum of 2 consecutive visits were required to diagnose permanent $\mathrm{AF}$ with no interim documented sinus rhythm or further evidence of sinus rhythm until the end of the study or the patient's death. Moreover, it was a prerequisite that the DDD mode was reprogrammed to ventricular-based pacing prior to, or at the end of follow-up. The time of permanent AF onset was defined as the first instance when $\mathrm{AF}$ was detected after implant and at the end of follow-up fulfilled the criteria of minimum duration, no evidence of sinus rhythm in the interim and pacing mode reprogramming from the DDD mode to the ventricular-based one.

Statistical analysis Data were analyzed using IBM SPSS Statistics Version 25.0 software (IBM Corp, Armonk, New York, United States). Continuous variables are expressed as mean (SD), and median (interquartile range [IQR]) if not normally distributed. Normal distribution was tested with the Kolmogorow-Smirnow test with the Lilliefors correction. Continuous variables were compared by means of the MannWhitney test. Categorical variables were compared using the Pearson $\chi^{2}$ test. The proportion of patients who progressed to permanent AF was calculated by the Kaplan-Meier method. Mortality rates between the study cohort and patients lost to follow-up were compared with log-rank test. Proportional hazards Cox regression analysis was used to determine the association between selected baseline variables and permanent AF development. We assessed the effect of time-covariate interaction to test the validity of the proportional hazards. Cox regression with time-dependent covariate was used to assess the association between permanent $\mathrm{AF}$ occurrence and post-implant survival and subsequently to explore potential subgroup effects. ${ }^{16} \mathrm{~A} P$ value of less than 0.05 was considered significant.

RESULTS Between October 4, 1984 and December 31, 2014 a total of 3932 consecutive patients underwent de novo DDD PPM implantation. 
TABLE 1 Comparison of patients with and without postdischarge follow-up

\begin{tabular}{|c|c|c|c|}
\hline Parameter & $\begin{array}{l}\text { Lost to follow-up } \\
\qquad(n=157[4 \%])\end{array}$ & $\begin{array}{l}\text { Remained } \\
\text { in follow-up } \\
(n=3771[96 \%])\end{array}$ & Pvalue \\
\hline $\begin{array}{l}\text { Age at implantation, y, median } \\
\text { (IQR) }\end{array}$ & $71.8(13.9)$ & $71.9(14.2)$ & 0.85 \\
\hline Female sex & $56(36)$ & $1761(47)$ & 0.007 \\
\hline SSS indication & $83(53)$ & $2527(67)$ & $<0.001$ \\
\hline Preimplant AF & $42(27)$ & $1276(34)$ & 0.07 \\
\hline RV lead at apex & $68(43)$ & $1625(43)$ & 0.96 \\
\hline All-cause mortality & $93(59)$ & $1342(36)$ & $<0.001$ \\
\hline
\end{tabular}

Data are presented as number (percentage) of patients unless otherwise indicated.

Abbreviations: AF, atrial fibrillation; IQR, interquartile range; RV, right ventricular; SSS, sick sinus syndrome

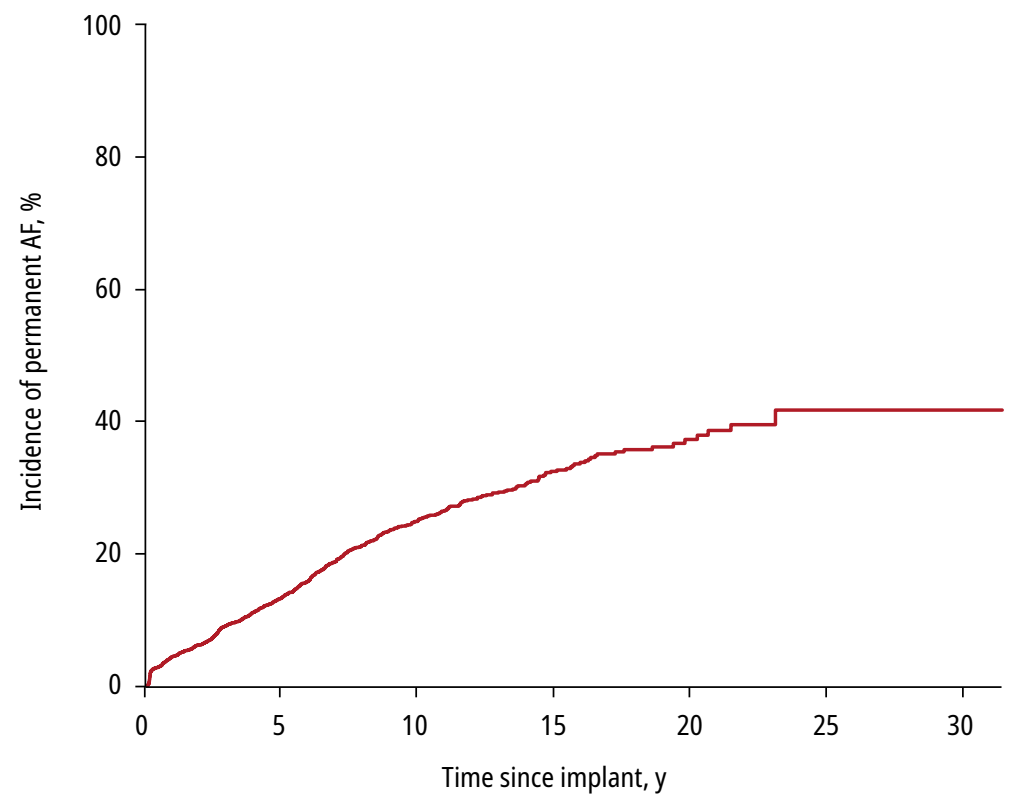

FIGURE 1 Kaplan-Meier curve showing time to occurrence of permanent atrial fibrillation (AF) during the study period

TABLE 2 Comparison of baseline characteristics in patients with and without permanent atrial fibrillation in the entire cohort

\begin{tabular}{llll} 
Parameter & $\begin{array}{c}\text { Permanent AF } \\
(\mathrm{n}=717[19 \%])\end{array}$ & $\begin{array}{c}\text { No permanent AF } \\
(\mathrm{n}=3054[81 \%])\end{array}$ & P value \\
\hline $\begin{array}{l}\text { Age at implantation, y, median } \\
(\mathrm{IQR})\end{array}$ & $71.9(11.7)$ & $71.9(15.1)$ & 0.05 \\
\hline Female sex & $344(48)$ & $1417(52)$ & 0.45 \\
\hline SSS indication & $551(77)$ & $1976(23)$ & $<0.001$ \\
\hline Preimplant AF & $408(57)$ & $868(28)$ & $<0.001$ \\
\hline RV lead at apex & $402(56)$ & $1223(44)$ & $<0.001$ \\
\hline
\end{tabular}

Data are presented as number (percentage) unless otherwise indicated.

Abbreviations: see TABLE 1

We excluded 4 patients whose vital status as of the end of follow-up was unknown and 157 patients who did not attend pacemaker clinic during follow-up. Comparison of patients who were lost to follow-up after initial DDD PPM implantation with patients who remained in follow-up is presented in TABLE 1 .

The remaining 3771 patients and a total of 24431.8 patient-years of follow-up were analyzed. Women constituted $46.7 \%$ of patients. Mean (SD) device follow-up was 6.5 (5.2) years, (median [IQR], 5.2 [6.5] years; maximal follow-up, 30.8 years). In total, we analyzed 29581 postimplant encounters in which device parameters, electrogram, and ECG were obtained (mean [SD], 7.8 [6.4]; median [IQR], 5 [8]). The mean (SD) survival time (eg, until patients' death or study follow-up completion) was 7.8 (5.3) years (median [IQR], 6.5 [6.8] years; maximal survival time, 31.3 years). AF prior to DDD pacemaker implantation was detected in 1276 (34\%) patients. Patients with preexisting AF compared with patients without AF were older (mean [SD] age at implantation, 71.8 [10] years; median [IQR], 73 [11.9] years vs mean [SD], 68.8 [12.9] years; median [IQR], 71.3 [15.3] years, respectively; $P<0.001$ ), more frequently female ( $56 \%$ vs $42 \%$, respectively; $P<0.001)$, and had higher prevalence of SSS relative to AVB ( $84 \%$ vs $58 \%$, respectively; $P<0.001$ ).

During the entire follow-up, 717 patients (19\%) developed permanent AF in a mean (SD) period of 4.6 (4.1) years (median [IQR], 3.6 [5.4] years). At year 1, 5, 10, and 15 after implant, 4.4\%, $13.3 \%, 25 \%, 32.3 \%$ of patients had permanent AF, respectively (FIGURE 1). Comparison of patients with permanent $A F$ versus patients without permanent $\mathrm{AF}$ is presented in TABLE 2. Additionally, the comparison of patients who underwent DDD PPM during the last decade of the inclusion period (2005-2014) assessing differences in baseline characteristics between patients who developed permanent AF versus those with nonpermanent AF or in sinus rhythm is presented in TABLE3.

In the whole cohort, male sex was associated with significantly higher risk of permanent AF development compared with female sex (HR, 1.316; 95\% CI, 1.134-1.528; $P<0.001)$. Each 1-year increase in age at implantation (HR, 1.041; 95\% CI, 1.033-1.049; $P<0.001)$ and history of AF (HR, 3.521; 95\% CI, 3.002-4.128; $P<0.001)$ were independent predictors for permanent AF occurrence. The position of the RV lead and pacing indication were not associated with permanent $\mathrm{AF}$ (FIGURE 2). The additional Cox regression analysis of 2475 patients who had DDD PPM implantation in the last decade replicated the findings of the entire cohort analysis (FIGURE 3 ).

A total of 310 patients (43\%) with permanent AF died after a mean (SD) period of 4.6 (3.6) years (median [IQR], 3.9 [5.5] years; maximum 17.3 years) following the onset of permanent AF. In the group without permanent $\mathrm{AF}, 1032 \mathrm{pa}-$ tients (32\%) died after mean (SD) 6.2 (5.0) years (median [IQR], 4.9 [6.7] years) following the first implantation. During the exposure to permanent $\mathrm{AF}$, the rate of death was 85 per every 1000 
TABLE 3 Comparison of baseline characteristics among patients who underwent implantation from 2005 to 2014

\begin{tabular}{llll} 
Parameter & $\begin{array}{c}\text { Permanent AF } \\
(\mathrm{n}=368[15 \%])\end{array}$ & $\begin{array}{c}\text { No permanent AF } \\
(\mathrm{n}=2107[85 \%])\end{array}$ & P value \\
$\begin{array}{l}\text { Age at implantation, y, median } \\
(\mathrm{IQR})\end{array}$ & $75.5(11.1)$ & $73.9(13.4)$ & 0.003 \\
\hline Female & $178(48)$ & $1007(48)$ & 0.84 \\
\hline SSS indication & $288(78)$ & $1327(63)$ & $<0.001$ \\
\hline Preimplant AF & $255(69)$ & $703(33)$ & $<0.001$ \\
\hline RV lead at apex & $67(18)$ & $298(14)$ & 0.04 \\
\hline
\end{tabular}

Data are presented as number (percentage) unless otherwise indicated.

Abbreviations: see TABLE 1

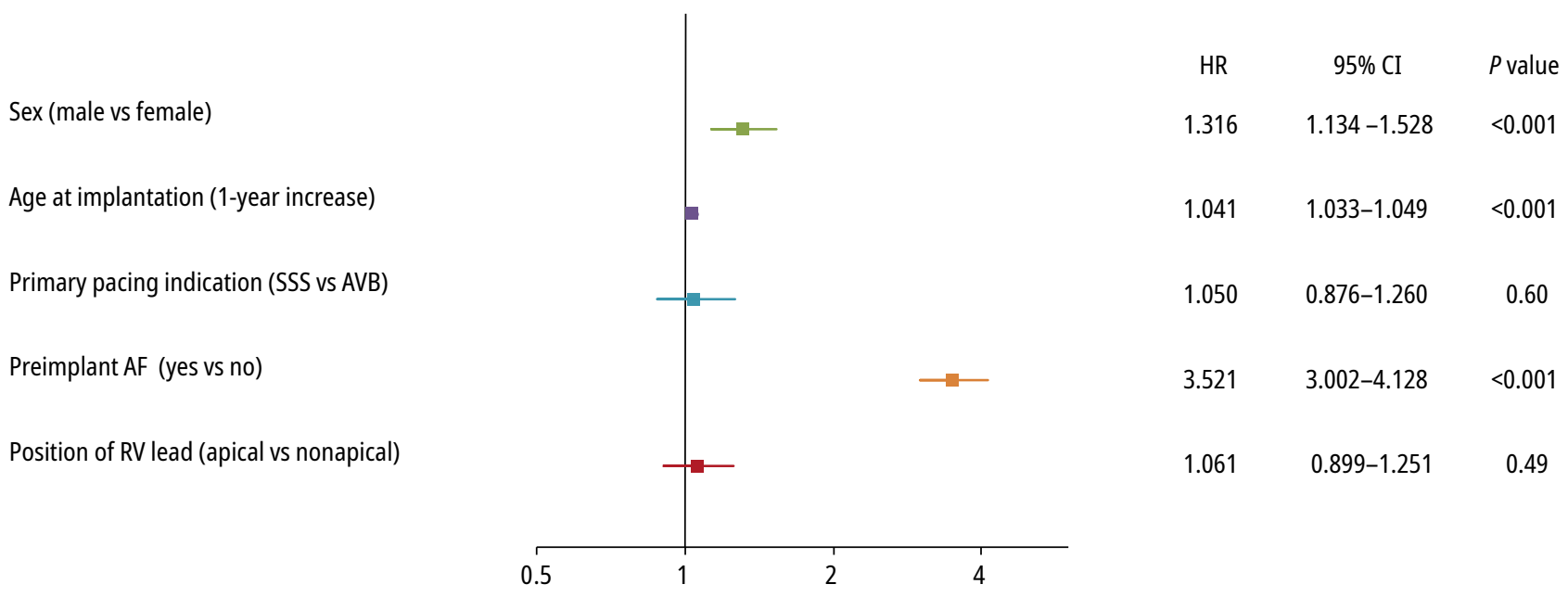

FIGURE 2 Association of baseline factors with permanent atrial fibrillation after dual-chamber pacemaker implantation in the entire cohort

Abbreviations: AVB, atrioventricular block; HR, hazard ratio; others, see TABLE 1

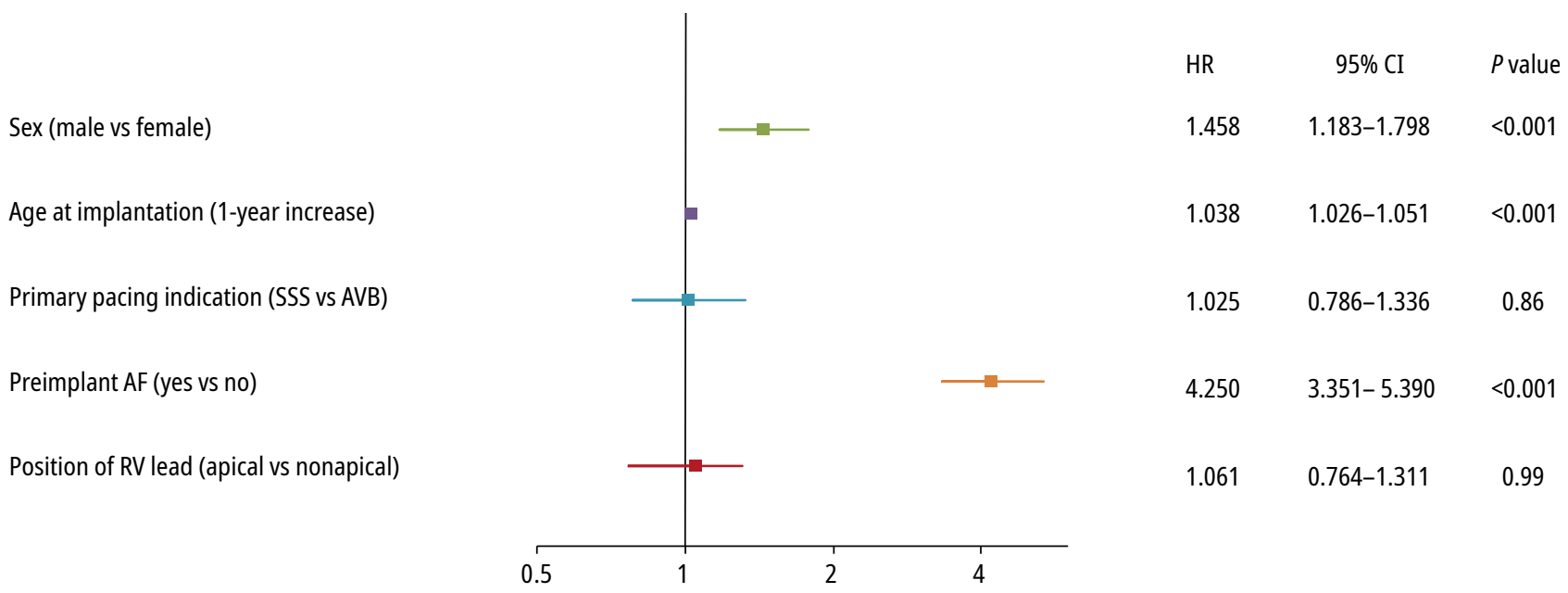

FIGURE 3 Association of baseline factors with permanent atrial fibrillation in group of patients who underwent implantation between 2005-2014 patient-years, whereas during nonexposure to permanent $\mathrm{AF}$, the rate of death was 40 per every 1000 patient-years of the study. Cox proportional hazards model with time-varying covariate confirmed that occurrence of permanent $\mathrm{AF}$ significantly increased mortality during follow-up (HR, 1.885; 95\% CI, 1.654-2.148; $P<0.001$ ). The observed effect remained significant after adjustment for age at implantation and sex (HR, 1.475; 95\% CI, 1.294-1.682; $P<0.001$ ). The significant association was consistently observed in patients selected with regard to age ( $>70$ years: $\mathrm{HR}$, 2.15 ; $\leq 70$ years: HR, 1.403), sex (men: HR, 1.841; women: HR, 1.932), index arrhythmia (AVB: HR, 2.190; SSS: HR, 1.796), history of AF (yes: HR, 1.401; no: HR, 2.486), and position of the RV lead (apical: HR, 1.924; nonapical: HR, 1.768).

Abbreviations: see TABLE 1 and FIGURE 2 
DISCUSSION Epidemiology of permanent atrial fibrillation in patients with a dual-chamber pacemaker This study demonstrates that the incidence of permanent AF development during a mean 6.5 years of follow-up reached $19 \%$. The percentage of patients with preimplant $\mathrm{AF}$ at baseline amounted to $34 \%$. Our findings accord with previous prospective and retrospective reports. In paced populations, the incidence of chronic AF was several times higher than in nonpaced patients and could be estimated at $1.6 \%$ to $3.8 \%$ per year, and from $15 \%$ to $20 \%$ as a cumulative lifetime incidence. ${ }^{17,18}$ In prospective analyses undertaken predominantly in patients with sinus node dysfunction implanted with DDD PPM, the rate of preimplant AF was between $36 \%$ and $63 \%,{ }^{17,19-21}$ whereas the incidence of permanent AF was estimated at $8 \%$ to $15.2 \%$ after a mean period of follow-up from 1.7 to 5.4 years. ${ }^{17,19-22}$ Skanes et $\mathrm{l}^{23}$ reported in a prospective trial the rate of chronic AF in DDD pacemaker population at $2.8 \%$ per year. In retrospective studies analyzing permanent AF development, such an endpoint was noted in $6.4 \%$ to $22 \%$ of patients after a mean follow-up from 2.7 to 7 years. ${ }^{24-26}$ Noteworthy, Konieczyńska et $\mathrm{al}^{27}$ demonstrated alarmingly low awareness of arrhythmia symptoms and their progressive nature in patients with AF and with or without a cardiac device. Only approximately one-third of patients who were surveyed in a tertiary cardiology center perceived AF as a progressive disease and a similar proportion of patients knew that AF may not always be symptomatic. Other significant knowledge gaps were identified with regard to treatment and complications. Furthermore, authors found that knowledge of AF symptoms and its progressive course was significantly worse in patients from a small volume district hospital compared with peers from a tertiary cardiology center. ${ }^{28}$ Essential and relevant information may be provided to patients with $\mathrm{AF}$ and a pacemaker at regular device follow-up visits.

Risk factors for permanent atrial fibrillation In terms of sex, prevalence and incidence rates of $\mathrm{AF}$ are higher in men than in women regardless of age. ${ }^{1,8,29-31}$ In 2010, the prevalence rates were 596 in men and 373 in women, and incidence rates were 76 in men and 60 in women, per 100000 people. ${ }^{30}$ After adjustment for age and other risk factors, men had a 1.5 to 2 times greater risk of developing AF than women. ${ }^{32,33}$ However, because women live longer than men and the prevalence of AF increases with age, the absolute number of women with $\mathrm{AF}$ exceeds that of men. Potpara et $\mathrm{al}^{34}$ reported that paroxysmal AF was more prevalent in women than in men at baseline, similarly to our results. Of note, recent meta-analysis including 6 large randomized controlled trials on non-vitamin $\mathrm{K}$ antagonist oral anticoagulation demonstrated that patients with paroxysmal AF were more likely to be women compared with patients with persistent or permanent AF. ${ }^{12}$ Surprisingly, we found that male sex was associated with increased risk of permanent AF development. Studies on nonpacemaker populations have not identified sex-related differences with regard to permanent AF. ${ }^{34-36}$ On the other hand, Veasey et a ${ }^{10}$ showed that in patients with DDD PPM and paroxysmal AF, male sex was significantly associated with progression to persistent or permanent AF in univariate model, but it was not found to be independently significant on multivariate analysis.

In the current study, age at baseline was associated with permanent $\mathrm{AF}$ development. It is well recognized that the incidence of AF increases with age. Age was demonstrated as independent factor for AF and chronic permanent AF in numerous previous studies. 5,8,22,25,29,31,35-38 The odds ratio of AF for each decade of advancing age was 2.1 for men and 2.2 for women. ${ }^{22}$

In our population, patients with a history of AF had more frequently SSS compared with AVB. It is consistent with the findings of Alonso et al, ${ }^{39}$ who estimated that the incidence rate of AF was 10-fold higher in patients with SSS compared with those without. In accordance with our findings, history of AF before pacemaker implantation was the strongest independent predictor of postimplant $\mathrm{AF}$ in both prospective ${ }^{17}$ and retrospective studies. ${ }^{25,26,37,38}$

To our knowledge, no randomized large-scale clinical studies have demonstrated so far a more deleterious clinical long-term effect of the RV apical pacing compared with septal pacing in bradycardia pacemaker recipients with normal baseline left ventricular function. A randomized trial comparing sustained apical RV pacing with septal pacing in patients with high-grade AVB failed to demonstrate any difference in $\mathrm{AF}$ burden over a 2-year study period. ${ }^{40}$ Of note, a retrospective study involving 477 consecutive patients who underwent PPM implantation for complete or advanced AVB demonstrated that RV pacing in the Hisian area compared with RV pacing in apical and septal positions was associated with lower risk of persistent/permanent AF after mean follow-up of nearly 5 years. ${ }^{38}$ Importantly, the reported risk of chronic AF development was similar in both apical and septal RV pacing groups with the rate of progression at $25.7 \%$ and $28 \%$, respectively. ${ }^{38}$ In keeping with these findings, we found that the risk of permanent AF development was similar between patients with apical and nonapical RV lead position. Moreover, the analysis in patients who had a DDD PPM implanted during the last decade of the inclusion period showed similar results. 
Mortality In patient aged 55 to 94 years from the original cohort of the Framingham study, AF was independently associated with a 1.5 -fold and 1.9-fold increased risk of death in men and women, respectively. ${ }^{4}$ Consequently, AF limited the advantage that women have over men in regard to longevity. ${ }^{4}$ Other authors also observed that patients with AF had increased mortality risk as compared with those without $\mathrm{AF}$, even after accounting for other comorbidities. ${ }^{3,7} \mathrm{~Pa}$ tients with incident AF had a 3.5-fold higher risk of death compared with those without $\mathrm{AF}$ according to Magnussen et al. ${ }^{5}$ Interestingly, in our previous analysis on the association between baseline factors and long-term mortality, we did not find significantly increased mortality in patients with a DDD PPM and preimplant AF compared with patients without history of $\mathrm{AF}$ at baseline. ${ }^{9} \mathrm{On}$ the other hand, in the present study, we confirmed that permanent $\mathrm{AF}$ was associated with unfavorable outcomes in terms of long-term survival. Our results are in line with the analysis by Polewczyk et $\mathrm{al}^{41}$ who showed that permanent AF was associated with significantly higher mortality following transvenous lead extraction in the mean follow-up of 3.7 years. Additionally, our findings are in line with recent reports on the general population showing that permanent AF was a predictive factor for mortality compared with paroxysmal AF. In a real-world community-based cohort study, the progression to permanent $\mathrm{AF}$ was an independent risk factor for death or hospital admission. ${ }^{11}$ An observation that patients with persistent or permanent AF have significantly higher risk of all-cause mortality compared with those with paroxysmal $\mathrm{AF}$ was validated in the recent meta-analysis involving 6 large-scale randomized controlled trials on oral anticoagulation therapy and a total of 70447 patients with AF. ${ }^{12}$

Study limitations Our observations should be interpreted in the context of limitations imposed by a retrospective study design. We did not take into account patients' comorbidities, medications, baseline echocardiographic parameters at index procedure and we did not track subsequent conducted cardioversions, AF ablations, and changes during follow-up in incident comorbidities, medicine prescribing, echocardiographic parameters, and device reprogramming, all of which might have influenced the permanent AF occurrence. The primary reason was the missing data due to patients' cardiology follow-up in local centers and destruction of paper patient records following a 20-year retention period. Additionally, clinical risk factors are dynamic in nature, for instance, elderly patients accumulate comorbidities, atrial and ventricular pacing percentage is frequently changing depending on device programming and medications. In the light of the above considerations, we chose to base our analysis on static baseline risk factors and complete data for the entire population.

Another limitation was the lack of continuous cardiac rhythm monitoring. The diagnosis of permanent AF was mostly based on standard intermittent monitoring techniques during pacemaker checks. Pacemakers equipped with automated storage of intracardiac electrocardiograms and AF diagnostic capabilities were not available for more than half of the 30-year study enrolment period. Hence, it is plausible that a considerable percentage of our patients with conventionally defined permanent AF might have spontaneously reverted into sinus rhythm between yearly follow-up visits and, in fact, remained in paroxysmal or persistent form of AF. ${ }^{10,42}$

Conclusions Our data reveal several important findings, such as the increased risk of permanent $\mathrm{AF}$ development in men, and confirmed the association with increasing age and history of preimplant AF. On the other hand, the position of the RV lead and type of pacing indication were not related to permanent AF. Importantly, these results remained unchanged when only the last decade of implantation period was analyzed. Furthermore, the current study showed increased mortality rates in patients with permanent AF compared with those without permanent AF.

\section{ARTICLE INFORMATION}

\section{CONFLICT OF INTEREST None declared.}

OPEN ACCESS This is an Open Access article distributed under the terms of the Creative Commons Attribution-NonCommercial-NoDerivatives 4.0 International License (CC BY-NC-ND 4.0), allowing third parties to download articles and share them with others, provided the original work is properly cited, not changed in any way, distributed under the same license, and used for noncommercial purposes only. For commercial use, please contact the journal office at kardiologiapolska@ptkardio.pl.

HOW TO CITE Dębski M, Ulman M, Ząbek A, et al. Permanent atrial fibrillation in patients with a dual-chamber pacemaker. Kardiol Pol. 2019; 77: 1140-1146. doi:10.33963/KP.14974

\section{REFERENCES}

1 Haim M, Hoshen M, Reges 0, et al. Prospective national study of the prevalence, incidence, management and outcome of a large contemporary cohort of patients with incident non-valvular atrial fibrillation. J Am Heart Assoc. 2015; 4: e001486.

2 Kirchhof P, Benussi S, Kotecha D, et al. 2016 ESC Guidelines for the management of atrial fibrillation developed in collaboration with EACTS. Eur Heart J. 2016; 37: 2893-2962.

3 Andersson T, Magnuson A, Bryngelsson IL, et al. Patients without comorbidities at the time of diagnosis of atrial fibrillation: causes of death during long-term follow-up compared to matched controls. Clin Cardiol. 2017; 40: 1076-1082.

4 Benjamin EJ, Wolf PA, D'Agostino RB, et al. Impact of atrial fibrillation on the risk of death: the Framingham Heart Study. Circulation. 1998; 98: 946-952.

5 Magnussen C, Niiranen TJ, 0jeda FM, et al. Sex differences and similarities in atrial fibrillation epidemiology, risk factors, and mortality in community cohorts: results from the BiomarCaRE consortium (Biomarker for Cardiovascular Risk Assessment in Europe). Circulation. 2017; 136: 1588-1597.

6 McIntyre WF, John PDS, Torabi M, Tate RB. Lifetime pattern of atrial fibrillation and the risks of stroke and death in a population-based cohort of men (from The Manitoba Follow-Up Study). Am J Cardiol. 2018; 122: 1688-1693.

7 Stewart S, Hart CL, Hole DJ, McMurray JJ. A population-based study of the long-term risks associated with atrial fibrillation: 20-year follow-up of the Renfrew/ Paisley study. Am J Med. 2002; 113: 359-364.

8 Vermond RA, Geelhoed B, Verweij N, et al. Incidence of atrial fibrillation and relationship with cardiovascular events, heart failure, and mortality: 
a community-based study from the Netherlands. J Am Coll Cardiol. 2015; 66: 1000-1007.

9 Dębski M, Ulman M, Ząbek A, et al. Association of selected factors with long-term prognosis and mortality after dual-chamber pacemaker implant. Cardiol J. 2018 Aug 29. [Epub ahead of print]

10 Veasey RA, Sugihara C, Sandhu K, et al. The natural history of atrial fibrillation in patients with permanent pacemakers: is atrial fibrillation a progressive disease? J Interv Card Electrophysiol. 2015; 44: 23-30.

11 Vidal-Perez R, Otero-Raviña F, Lado-López M, et al. The change in the atrial fibrillation type as a prognosis marker in a community study: long-term data from AFBAR (Atrial Fibrillation in the BARbanza) study. Int J Cardiol. 2013; 168: 2146-2152.

12 Zhang W, Xiong Y, Yu L, et al. Meta-analysis of stroke and bleeding risk in patients with various atrial fibrillation patterns receiving oral anticoagulants. Am J Cardiol. 2019; 123: 922-928.

13 Veasey RA, Arya A, Silberbauer ], et al. The relationship between right ventricular pacing and atrial fibrillation burden and disease progression in patients with paroxysmal atrial fibrillation: the long-MinVPACE study. Europace. 2011; 13: 815-820.

14 Dębski M, Maniecka-Bryła I, Dziankowska-Zaborszczyk E, et al. Years of life lost as a measure of premature death among dual-chamber pacemaker recipients from Małopolska Province. Kardiol Pol. 2019; 77: 683-687.

15 Dębski M, Ulman M, Ząbek A, et al. Lead-related complications after DDD pacemaker implantation. Kardiol Pol. 2018; 76: 1224-1231.

16 van Walraven C, Davis D, Forster AJ, Wells GA. Time-dependent bias was common in survival analyses published in leading clinical journals. J Clin Epidemiol. 2004; 57: 672-682.

17 Gillis AM, Morck M. Atrial fibrillation after DDDR pacemaker implantation. J Cardiovasc Electrophysiol. 2002; 13: 542-547.

18 Nielsen JC. Mortality and incidence of atrial fibrillation in paced patients. J Cardiovasc Electrophysiol. 2002; 13 (1 suppl): S17-S22.

19 Lamas GA, Lee KL, Sweeney M0, et al. Ventricular pacing or dual-chamber pacing for sinus-node dysfunction. N Engl J Med. 2002; 346: 1854-1862.

20 Nielsen JC, Thomsen PE, Højberg S, et al. A comparison of single-lead atrial pacing with dual-chamber pacing in sick sinus syndrome. Eur Heart J. 2011; 32: 686-696. 21 Sweeney M0, Bank AJ, Nsah E, et al. Minimizing ventricular pacing to reduce atrial fibrillation in sinus-node disease. N Engl J Med. 2007; 357: 1000-1008.

22 Boriani G, Tukkie R, Manolis AS, et al. Atrial antitachycardia pacing and managed ventricular pacing in bradycardia patients with paroxysmal or persistent atrial tachyarrhythmias: the MINERVA randomized multicentre international trial. Eur Heart J. 2014; 35: 2352-2362.

23 Skanes $A C$, Krahn AD, Yee R, et al. Progression to chronic atrial fibrillation after pacing: the Canadian Trial of Physiologic Pacing. J Am Coll Cardiol. 2001; 38: 167-172.

24 Ibrahim B, Sanderson JE, Wright B, Palmer R. Dual chamber pacing: how many patients remain in DDD mode over the long term? Br Heart J. 1995; 74: 76-79. 25 Sgarbossa EB, Pinski SL, Maloney JD, et al. Chronic atrial fibrillation and stroke in paced patients with sick sinus syndrome. Relevance of clinical characteristics and pacing modalities. Circulation. 1993; 88: 1045-1053.

26 Tse HF, Lau CP. Prevalence and clinical implications of atrial fibrillation episodes detected by pacemaker in patients with sick sinus syndrome. Heart. 2005; 91: 362-364.

27 Konieczyńska M, Sobieraj E, Bryk AH, et al. Differences in knowledge among patients with atrial fibrillation receiving non-vitamin $\mathrm{K}$ antagonist oral anticoagulants and vitamin K antagonists. Kardiol Pol. 2018; 76: 1089-1096.

28 Janion-Sadowska A, Sadowski M, Konieczyńska M, et al. Polish regional differences in patient knowledge on atrial fibrillation and its management as well as in patterns of oral anticoagulant prescription. Kardiol Pol. 2019; 77: 437-444.

29 Bose A, O'Neal WT, Wu C, et al. Sex differences in risk factors for incident atrial fibrillation (from the Reasons for Geographic and Racial Differences in Stroke [REGARDS] Study). Am J Cardiol. 2019; 123: 1453-1457.

30 Chugh SS, Havmoeller R, Narayanan K, et al. Worldwide epidemiology of atrial fibrillation: a Global Burden of Disease 2010 study. Circulation. 2014; 129: 837-847.

31 Miyasaka Y, Barnes ME, Gersh BJ, et al. Secular trends in incidence of atrial fibrillation in Olmsted County, Minnesota, 1980 to 2000, and implications on the projections for future prevalence. Circulation. 2006; 114: 119-125.

32 Benjamin EJ, Levy D, Vaziri SM, et al. Independent risk factors for atrial fibrillation in a population-based cohort. The Framingham Heart Study. JAMA. 1994; 271: 840-844.

33 Ko D, Rahman F, Schnabel RB, et al. Atrial fibrillation in women: epidemiology, pathophysiology, presentation, and prognosis. Nat Rev Cardiol. 2016; 13: 321-332.

34 Potpara TS, Marinkovic JM, Polovina MM, et al. Gender-related differences in presentation, treatment and long-term outcome in patients with first-diagnosed atrial fibrillation and structurally normal heart: the Belgrade atrial fibrillation study. Int J Cardiol. 2012; 161: 39-44.

35 Jahangir A, Lee V, Friedman PA, et al. Long-term progression and outcomes with aging in patients with lone atrial fibrillation: a 30-year follow-up study. Circulation. 2007; 115: 3050-3056.

36 Kerr CR, Humphries KH, Talajic M, et al. Progression to chronic atrial fibrillation after the initial diagnosis of paroxysmal atrial fibrillation: results from the Canadian Registry of Atrial Fibrillation. Am Heart J. 2005; 149: 489-496.
37 Al-Khatib SM, Wilkinson WE, Sanders LL, et al. Observations on the transition from intermittent to permanent atrial fibrillation. Am Heart J. 2000; 140: 142-145. 38 Pastore $\mathrm{G}$, Zanon F, Baracca E, et al. The risk of atrial fibrillation during right ventricular pacing. Europace. 2016; 18: 353-358.

39 Alonso A, Jensen PN, Lopez FL, et al. Association of sick sinus syndrome with incident cardiovascular disease and mortality: the Atherosclerosis Risk in Communities study and Cardiovascular Health Study. PLoS One. 2014; 9: e109662.

40 Kaye GC, Linker NJ, Marwick TH, et al. Effect of right ventricular pacing lead site on left ventricular function in patients with high-grade atrioventricular block: results of the Protect-Pace study. Eur Heart J. 2015; 36: 856-862.

41 Polewczyk A, Jacheć W, Polewczyk AM, et al. Infectious complications in patients with cardiac implantable electronic devices: risk factors, prevention, and prognosis. Pol Arch Intern Med. 2017; 127: 597-607.

42 Sugihara C, Veasey R, Freemantle N, et al. The development of AF over time in patients with permanent pacemakers: objective assessment with pacemaker diagnostics demonstrates distinct patterns of AF. Europace. 2015; 17: 864-870. 